\title{
MODAL PRECESSION OF A ROTATING HEMISPHERICAL SHELL
}

\author{
C. O. CHANG, J. J. HWANG and C. S. CHOU \\ Institute of Applied Mechanics, National Taiwan University, Taipei 10764, \\ Taiwan, P.R. China
}

(Received 9 December 1994; in revised form 27 July 1995)

\begin{abstract}
The purpose of this paper is to investigate the precession of vibrational modes (or standing waves) of a hemispherical shell due to the effect of a small constant axial rotation, where previously resonant modes exists. Niordson thin shell theory, which allows the stretch of the middle surface and is expressed in an invariant form, is employed to derive the equations of bending vibration of a rotating open shell. Analysis is divided into two phases. For the first one, only the Coriolis force is considered. The frequency change due to the presence of Coriolis force can be obtained by solving the equation of solvability condition in the course of perturbation analysis. The precession rate of the vibrational modes is obtained in an analytical expression. For the second phase both the Coriolis and centrifugal forces are considered. The displacements are considered as the vectorial sum of the initial and incremental displacements. The initial displacements result from the non-vibrational rotating shell which experiences centrifugal forces. The incremental displacements are the vibrational displacements of the rotating shell relative to the initial state. Results through analytical analysis show that the centrifugal force affects the frequencies of forward and backward travelling waves but does not influence the precession rate of the vibrational modes. Copyright $\mathbb{C} 1996$ Elsevier Science Ltd.
\end{abstract}

\section{INTRODUCTION}

Bryan (1890) was the first to investigate the dynamics of the revolving ring or cylinder, and discovered that Coriolis forces give rise to a splitting of the frequencies of natural modes of flexural vibrations, leading to precession of standing waves and beats. Since then, many people steadily improved our knowledge of rotating rings and cylinders [see, e.g., Carrier (1945), Srinivasan and Lauterbach (1971), Zhuravlev and Klimov (1983), Saito and Endo (1986), Huang and Soedel (1988)]. Shevchuk and Thullen (1978) experimentally produced the travelling wave phenomena on a rotating cylindrical shell by means of a stationary exciting force. Endo et al. (1984) experimentally measured the frequencies of forward and backward flexural travelling waves of a thin rotating ring.

Recently Matthews and Rybak (1992) commented that solid state gyroscopes, in which a vibrating hemispherical shell is used as the sensing element, become attractive and competitive in comparison with the ring laser gyroscopes or fibre optical gyroscopes due to the nature of optimum cost versus degree of required accuracy and reliability. Investigations of free vibrations of non-rotating spherical shells are numerous, but there are few about the case of rotating ones. Beckemyer and Eversman (1971) studied the effects of shell geometry parameters on the natural frequencies of free vibration of a spinning, centrally clamped, shallow spherical shell. Chen et al. (1993) analysed, by using the finite element method, the effects of inertial forces on the resonance frequencies of high speed rotating shells of revolution. None of them have investigated the precessional phenomenon of modal vibration patterns. The work of Loper and Lynch (1983) described a hemispherical resonator gyroscope and measured the precession rate of the vibrational mode. Both works of Loper and Lynch (1983) and Matthews and Rybak (1992) do not give theoretical verification.

Zhuravlev and Popov (1985) investigated the vibrations of a rotating hemispherical shell for the cases of both constant and time-varying spin rate. They proved that the precession of the standing wave is an integrating phenomenon. Their equations for the precession rate were obtained based on the use of the Rayleigh inextensional mode and the effect of the centrifugal force was not considered. Egarmin (1986) estimated the precession rate of a rotating axisymmetric shell by using the reciprocity theorem in a non-rigorous 
manner. To simplify the analysis he equated the displacements of two different deformed states corresponding to the two different rotating speeds of the shell, which are used in the reciporcity theorem, and approximated them by the displacement of non-rotating state. Centrifugal forces were not considered and inextensional middle surfaces were assumed. In this paper, instead of using the above-mentioned non-rigorous approach, the vibrational equations are solved directly by analytical methods to obtain the explicit expression for precession rate.

The assumption of inextensional middle surface, made in Lord Rayleigh's thin shell theory and adopted by Egarmin (1986), is removed in this paper. The reasons for this removal are as follows: Love (1888) pointed out that boundary conditions at a free edge of an open shell could not be satisfied without at least some extension of the middle surface. Niordson (1985) showed that for the thin open spherical shell case, Lord Rayleigh's solution gives an upper bound for the frequency and gives frequency of infinite value when the open shell becomes close, which is not true.

In this paper we make an attempt to derive an analytic expression for the precession rate of a rotating open spherical shell. Niordson's shell theory, which included the extensional deformation of the middle surface and was derived in a coordinate-independent form, is employed and extended to accommodate the inertial forces caused by the rotation of the shell. Analysis is divided into two phases. For the first phase, only the Coriolis force is considered, since the precession phenomenon of standing waves is primarily caused by it. For the second phase, the centrifugal force is added and its effect is analysed. Since the angular velocity of the vehicle measured by gyroscopes is usually small (below $1 \mathrm{~Hz}$ in most use), it is, therefore, assumed that the shell considered rotates at low speed $\Omega$ and the ratio $\varepsilon$ of $\Omega$ to the frequency of the first bending mode of the shell is small. For the first phase the response due to the effect of Coriolis force is considered as a perturbed solution with respect to the solution of free vibration of the non-rotating shell. Niordson (1984) and Kalnins and Wilkinson (1965) had obtained, respectively, the exact solutions of displacements in different approach for the non-rotating shell in free vibration. The unperturbed system is self-adjoint and exactly the same as the homogeneous part of the perturbed system. The perturbed frequencies are obtained by solving the equation of solvability condition which must be satisfied by the solution of perturbed system. Niordson's solution (1984) of free vibration of a non-rotating shell is directly taken as the solution of the unperturbed system which is needed in the equation of solvability condition. The analytical expression for the precession rate of the vibrational modes can be obtained without knowing the solution of the perturbed system. The result of precessional angle agrees well with the experimental one.

For the second phase the deformation displacements are decomposed into the initial and incremental ones. The initial displacements are the ones of the non-vibrating shell, which are caused by the centrifugal force. The incremental displacements are due to the vibration of the rotating shell and are measured with respect to the initial state. The initial displacements are obtained by solving the membrane equations. The terms, that are neglected in the equations of equilibrium, are shown to be small, which justifies the good approximation of the true state by the membrane state. Incremental equations of motion are derived by using the principle of virtual work. The method of travelling waves is adopted to seperate the time and circumferential variables from the latitudinal one. The application of Galerkin method to the resultant ordinary differential equations leads to the algebraic equations for natural frequencies $\omega$ of a rotating shell. These frequencies are expanded in series of $\varepsilon$. The second-order approximation of frequencies, which are obtained in explicit form, gives the answer about the effect of the centrifugal force on the wave spced of forward and backward travelling waves and the precession rate of the standing waves.

THE EFFECT OF CORIOLIS FORCE

\section{Equations of motion}

A thin hemispherical shell of radius $R$ and thickness $h$ clamped at the pole and rotating about the symmetric $Z$ axis at a constant speed $\Omega$ is shown in Fig. 1, wherc the $X, Y, Z$ 


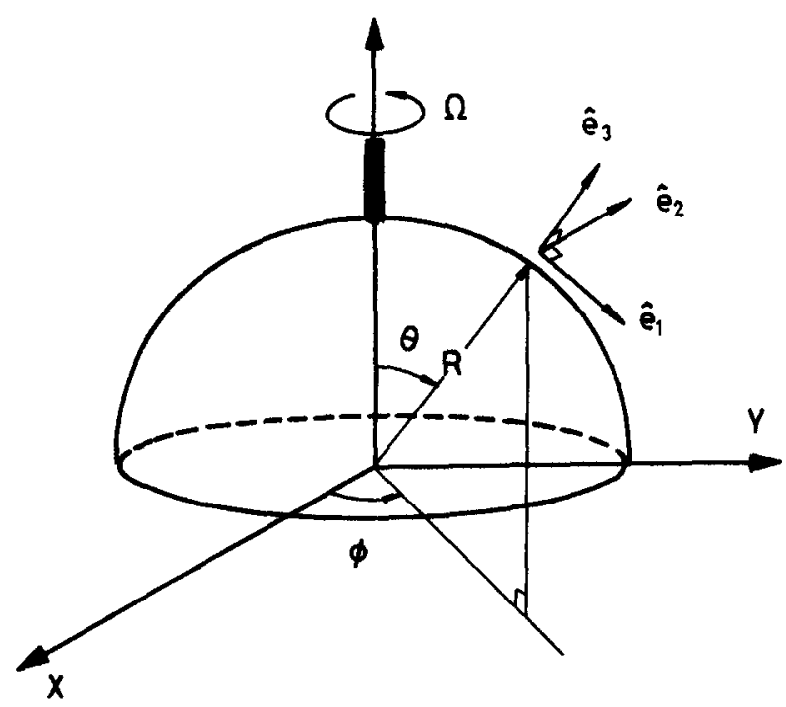

Fig. 1. Coordinate system.

axes are fixed in space. The middle surface of a shell in the undeformed state is parameterised by any two independent coordinates $\theta^{1}, \theta^{2}$. Let $\mathbf{a}_{\alpha}(\alpha=1,2)$ denote the covariant tangent vectors of the $\theta^{1}, \theta^{2}$ curvilinear coordinate system and $\mathbf{a}_{3}$ the unit normal vector to the shell. $\mathbf{a}_{3}$ is defined by $\mathbf{a}_{3}=\frac{1}{2} \varepsilon^{\alpha \beta} \mathbf{a}_{\alpha} \times \mathbf{a}_{\beta}$, where $\varepsilon^{\alpha \beta}$ is the two-dimensional permutation tensor. The metric tensor and curvature tensor are denoted by $a_{\alpha \beta}$ and $d_{\alpha \beta}$, respectively. For spherical shell $d_{\alpha \beta}=-(1 / R) a_{\alpha \beta}$. The displacement vector $\mathbf{u}$ of the middle surface due to deformation is denoted by $\mathbf{u}=u^{\alpha} \mathbf{a}_{\alpha}+w \mathbf{a}_{3}=u_{\beta} \mathbf{a}^{\beta}+w \mathbf{a}^{3}$. The corresponding physical components of displacements are $u=u^{1} / \sqrt{a^{11}}, v=u^{2} / \sqrt{a^{22}}$, and $w$, where $a^{11}=\mathbf{a}^{1} \cdot \mathbf{a}^{1}$ and $a^{22}=\mathbf{a}^{2} \cdot \mathbf{a}^{2}$. On the assumption of small strain and rotation the linear strain tensor $E_{\alpha \beta}$ and bending tensor $K_{\alpha \beta}$ take, respectively, the forms

$$
\begin{aligned}
& E_{\alpha \beta}=\frac{1}{2}\left(a_{\alpha \beta}^{*}-a_{\alpha \beta}\right) \cong \frac{1}{2}\left(D_{\alpha} u_{\beta}+D_{\beta} u_{\alpha}\right)+\frac{a_{\alpha \beta}}{R} w \\
& K_{\alpha \beta}=d_{\alpha \beta}^{*}-d_{\alpha \beta} \cong D_{\alpha} D_{\beta} w-\frac{1}{R}\left(D_{\alpha} u_{\beta}+D_{\beta} u_{\alpha}\right)-\frac{1}{R^{2}} a_{\alpha \beta} w,
\end{aligned}
$$

where quantities marked by the superscript '*' are measured in the deformed state and $D_{\alpha}$ denotes the covariant differentiation with respect to the surface coordinate $\theta^{\alpha}$. In order to get more simple equations of motion, an alternative bending tensor $\tilde{K}_{\alpha \beta}$ (Niordson, 1984) :

$$
\tilde{K}_{\alpha \beta}=K_{\alpha \beta}+\frac{2}{R} E_{\alpha \beta}=D_{\alpha} D_{\beta} w+\frac{1}{R^{2}} a_{\alpha \beta} w
$$

is used to replace $K_{\alpha \beta} . \tilde{K}_{\alpha \beta}$ is a function of $w$ only. Let $N^{\alpha \beta}$ and $M^{\alpha \beta}$ denote the membrane stress and moment tensors, respectively. Through the use of the principle of virtual work, $N^{\alpha \beta} \delta E_{\alpha \beta}+M^{\alpha \beta} \delta K_{\alpha \beta}=\tilde{N}^{\alpha \beta} \delta E_{\alpha \beta}+M^{\alpha \beta} \delta \tilde{K}_{\alpha \beta}$, the appropriate membrane stress tensor $\tilde{N}^{\alpha \beta}$ is obtained as $\tilde{N}^{\alpha \beta}=N^{\alpha \beta}-(2 / R) M^{\alpha \beta}$. The two-dimensional shell equations in terms of $M^{\alpha \beta}$ and $\tilde{N}^{\alpha \beta}$ are

$$
\begin{gathered}
D_{\alpha} \tilde{N}^{\alpha \beta}+F^{\beta}=0, \quad \beta=1,2 \\
D_{\alpha} D_{\beta} M^{\alpha \beta}+\frac{1}{R^{2}} M_{\alpha}^{\alpha}+\frac{1}{R} \tilde{N}_{\alpha}^{\alpha}-F^{3}=0,
\end{gathered}
$$


where $F^{\beta}$ and $F^{3}$ are components of resultant applied forces per unit area of the middle surface along surface coordinate system. Equations (4) and (5) are surface coordinatesindependent. The membrane stress and moment tensor, in tcrms of displacements based on Hooke's constitutive law, take the form:

$$
\tilde{N}^{\alpha \beta}=\frac{E h}{1-v^{2}}\left[(1-v)\left(\frac{1}{2} D_{\alpha} u_{\beta}+\frac{1}{2} D_{\beta} u_{\alpha}+\frac{1}{R} a_{\alpha \beta} w\right)+v a_{\alpha \beta}\left(D_{\gamma} u^{\gamma}+\frac{2 w}{R}\right)\right],
$$

and

$$
M^{\alpha \beta}=D\left[(1-v)\left(D^{\alpha} D^{\beta} w+\frac{1}{R^{2}} a^{\alpha \beta} w\right)+v a^{\alpha \beta}\left(\Delta+\frac{2}{R^{2}}\right) w\right]
$$

where $E$ and $v$ are the Young's modulus and Poisson ratio, respectively, $D=E h^{3} / 12\left(1-v^{2}\right)$, $D^{\alpha}=a^{\alpha \gamma} D_{\gamma}$, and $\Delta=D_{\alpha} D^{\alpha}$ is the Laplacian. If the spherical coordinates, $\theta^{1}=\theta$ and $\theta^{2}=\phi$, are chosen as the surface coordinates for the middle surface, then $a^{11}=1 / R^{2}$, $a^{22}=1 /\left(R^{2} \sin ^{2} \theta\right)$, and

$$
\Delta=\frac{1}{R^{2}}\left(\frac{\partial^{2}}{\partial \theta^{2}}+\cot \theta \frac{\partial}{\partial \theta}+\frac{1}{\sin ^{2} \theta} \frac{\partial^{2}}{\partial \phi^{2}}\right)
$$

The constant angular velocity of the shell expressed in the surface coordinate system is $\Omega=\Omega^{\prime} \mathbf{a}_{j}$ with components $\Omega^{1}=-\Omega \sin \theta / R, \Omega^{2}=0$, and $\Omega^{3}=\Omega \cos \theta$. The external forces in shell equations (4) and (5) are the inertial forces $-\rho h[\ddot{\mathbf{u}}+2 \Omega \times \dot{\mathbf{u}}]$, with the centrifugal force neglected due to the assumption of small rotating speed. Their components along the surface coordinate system are

$$
-F^{\beta}=\rho h \ddot{u}^{\beta}+2 \rho h \varepsilon^{\beta i j} \Omega_{i} \dot{u}_{j} ; \quad-F^{3}=\rho h \ddot{w}+2 \rho h \varepsilon^{3 \alpha \beta} \Omega_{\alpha} \dot{u}_{\beta} ; \quad \alpha, \beta=1,2, \quad i, j=1,2,3
$$

where $\rho$ is the density, $\varepsilon^{i j k}$ is the three-dimensional permutation tensor. The boundary conditions for the shell clamped at the pole are

$$
u=v=w=\frac{\partial w}{\partial \theta}=0, \text { at } \quad \theta=0
$$

and the boundary conditions for the free edge at $\theta=\pi / 2$

$$
\begin{aligned}
N & =\tilde{N}^{\alpha \beta} n_{\alpha} n_{\beta}=0, \quad S=\tilde{N}^{\alpha \beta} n_{\alpha} t_{\beta}=0, \\
M_{B} & =M^{\alpha \beta} n_{\alpha} n_{\beta}=0, \quad Q=-n_{\beta} D_{\alpha} M^{\alpha \beta}-\frac{\partial}{\partial s}\left(M^{\alpha \beta} n_{\alpha} t_{\beta}\right)=0,
\end{aligned}
$$

where $N$ and $S$ are the normal and tangent membrane stresses, respectively, acting on the boundary curve $\mathscr{L}$ of the middle surface, $M_{B}$ and $Q$ are the bending moment and effective shear force on $\mathscr{L}, n_{\alpha}$ and $t_{\alpha}$ are the components of unit outward normal and tangent vectors to $\mathscr{L}$, the variable $s$ in eqn (10d) represents the arc length of the boundary curve $\mathscr{L}$.

Analysis

The solutions for the displacements $u^{1}, u^{2}$, and $w$ are assumed in the travelling wave type as 


$$
\left\{\begin{array}{l}
u^{1} \\
u^{2} \\
w
\end{array}\right\}=\left\{\begin{array}{l}
\bar{u}^{1}(\theta) \cos (p \phi-\omega t) \\
\bar{u}^{2}(\theta) \sin (p \phi-\omega t) \\
\bar{w}(\theta) \cos (p \phi-\omega t)
\end{array}\right\},
$$

where $\omega$ is the wave speed of the travelling wave and $|\omega|$ represents the natural frequency of the rotating shell, $p$ is the number of circumferential wave, $\bar{u}^{1}, \bar{u}^{2}$, and $\bar{w}$ are the displacement components of an arbitrary, say the $n$ th, latitudinal mode. For simplicity the operators in eqns (4) and (5) are redefined as

$$
L^{\beta}(\mathbf{u})=D_{\alpha} \tilde{N}^{\alpha \beta}, \quad-L(\mathbf{u})=D_{\alpha} D_{\beta} M^{\alpha \beta}+\frac{1}{R^{2}} M_{\alpha}^{\alpha}+\frac{1}{R} \tilde{N}_{\alpha}^{\alpha}, \quad \alpha, \beta=1,2 .
$$

Substituting eqns (8) and (11) into eqns (4) and (5) and letting the coefficients of $\cos (p \phi-\omega t)$ and $\sin (p \phi-\omega t)$ in the left-hand side of resultant equations to those in the right-hand side, we obtain :

$$
\begin{aligned}
\overline{L^{1}(\mathbf{u})}+\rho h \omega^{2} \bar{u}^{1} & =2 \rho h \Omega \omega \overline{\bar{v}} \frac{\cos \theta}{R}, \\
\overline{L^{2}(\mathbf{u})}+\rho h \omega^{2} \bar{u}^{2} & =2 \rho h \omega \frac{\Omega}{R}(\bar{u} \cot \theta+\bar{w}), \\
\overline{L(\mathbf{u})}+\rho h \omega^{2} \bar{w} & =2 \rho h \Omega \omega \bar{v} \sin \theta .
\end{aligned}
$$

The overlined quantities in eqn (12) mean that they are functions of $\theta$ only, such as

$$
\overline{L^{1}(\mathbf{u})}=\frac{E h}{1-v^{2}} a^{11}\left[\frac{1-v}{2}\left(\bar{\Delta}+\frac{1}{R^{2}}\right) \bar{u}_{1}+\frac{1+v}{2} \overline{D_{1} D_{\delta} u^{\delta}}+\frac{1+v}{R} \overline{D_{1} w}\right]
$$

where

$$
\begin{aligned}
\bar{\Delta} & =\frac{1}{R^{2}}\left(\frac{\mathrm{d}^{2}}{\mathrm{~d} \theta^{2}}+\cot \theta \frac{\mathrm{d}}{\mathrm{d} \theta}-\frac{p^{2}}{\sin ^{2} \theta}\right), \\
\widetilde{D_{\gamma} u^{\gamma}} & =\widehat{u_{, \gamma}^{\gamma}+\Gamma_{\gamma \beta}^{\gamma} u^{\beta}}=\left[\bar{u}_{, \theta}^{1}+p \bar{u}^{2}+\cot \theta \bar{u}^{1}\right],
\end{aligned}
$$

$\Gamma_{i j}^{k}$ is Christoffel symbol of second kind, and

$$
\overline{D_{1} D_{\gamma} u^{y}}=\left[\bar{u}_{, \theta}^{1}+p \bar{u}^{2}+\cot \theta \bar{u}^{1}\right]_{, \theta}, \quad \overline{D_{1} w}=\bar{w}_{, \theta} .
$$

Let $\epsilon=\Omega / \omega_{0}$, where $\left|\omega_{0}\right|$ denotes the natural frequency of the $n$th latitudinal and the $p$ th circumferential mode of the non-rotating shell. $\epsilon$ is a small parameter on the assumption that $\Omega \ll\left|\omega_{0}\right|$. We consider that the solutions of eqn (12) consist of the unperturbed one, which is the response of the free vibration of the non-rotating shell, and the perturbed one, which is caused by the Coriolis acceleration due to the rotation. So the displacements and frequency are expanded in series of $\epsilon$ as

$$
\begin{aligned}
u^{\alpha} & =\stackrel{0}{u}^{\alpha}+\epsilon \mathfrak{u}^{\alpha}+\cdots, \quad \alpha=1,2 \\
w & =\stackrel{0}{w}+\epsilon \stackrel{1}{w}+\cdots, \\
\omega & =\omega_{0}+\epsilon \omega_{1}+\cdots .
\end{aligned}
$$


The system of eqns (4) and (5) is linear based on the assumption of small deformation, therefore, the stresses can also be expanded in series of $\epsilon$ in the same form of displacements

$$
N^{\alpha \beta}=\stackrel{0}{N^{\alpha \beta}}+\epsilon \stackrel{1}{N}^{\alpha \beta}+\cdots ; \quad M^{\alpha \beta}=\stackrel{0}{M}^{\alpha \beta}+\epsilon \stackrel{1}{M}^{\alpha \beta}+\cdots,
$$

Substituting eqn (13) into eqn (12) and setting the coefficients of like powers of $\epsilon$ equal zero, we obtain the governing equations for $\bar{u}^{1}, \bar{u}^{2}$, and $\frac{0}{\bar{w}}$

$$
\begin{aligned}
& \overline{L^{\alpha}(\overline{\mathbf{u}})}+\rho h \omega_{0}^{2} \frac{0}{\bar{u}^{\alpha}}=0, \\
& \overline{L(\mathbf{u})}+\rho h \omega_{0}^{2} \overline{\bar{w}}=0, \quad \alpha=1,2,
\end{aligned}
$$

and the governing equations for $\frac{1}{u^{1}}, \frac{1}{u^{2}}$, and $\frac{1}{w}$

$$
\begin{aligned}
& \overline{L^{1}(\mathbf{u})}+\rho h \omega_{0}^{2} \frac{1}{\bar{u}^{1}}=-2 \rho h \omega_{0} \omega_{1} \frac{\frac{0}{u}}{R}+2 \rho h \omega_{0}^{2} \frac{0}{\bar{v}} \frac{\cos \theta}{R}, \\
& \overline{L^{2}(\mathbf{u})}+\rho h \omega_{0}^{2} \frac{1}{\bar{u}^{2}}=-2 \rho h \omega_{0} \omega_{1} \frac{\frac{0}{\bar{v}}}{R \sin \theta}+2 \rho h \omega_{0}^{2}\left(\frac{0}{\bar{u}} \cos \theta+\frac{0}{\bar{w}} \sin \theta\right) \frac{1}{R \sin \theta}, \\
& \overline{L(\mathbf{u})}+\rho h \omega_{0}^{2} \frac{1}{\bar{w}}=-2 \rho h \omega_{0} \omega_{1} \frac{0}{\bar{w}}+2 \rho h \omega_{0}^{2} \frac{0}{\bar{v}} \sin \theta .
\end{aligned}
$$

Substituting eqn (14) into eqns (9) and (10), the clamped boundary conditions at $\theta=0^{\circ}$ become

$$
\stackrel{0}{\bar{u}}=\stackrel{0}{\bar{v}}=\stackrel{0}{\bar{w}}=\frac{\partial \overline{\bar{w}}}{\partial \theta}=0, \quad \frac{1}{\bar{u}}=\frac{1}{\bar{v}}=\frac{1}{\bar{w}}=\frac{\partial \frac{1}{\bar{w}}}{\partial \theta}=0,
$$

and the boundary conditions at $\theta=\pi / 2$ become

$$
\stackrel{0}{\bar{N}}=\stackrel{0}{\bar{S}}={\stackrel{0}{M_{B}}}_{B}=\stackrel{0}{\bar{Q}}=0, \quad \stackrel{1}{\bar{N}}=\stackrel{1}{\bar{S}}=\stackrel{1}{M}_{B}=\stackrel{1}{Q}=0 .
$$

Only the solutions of first approximation are considered here. As can be seen, the operators of the homogeneous part of eqns (16) are the same as those of eqns (15). It is well known that if a self-adjoint system, $L(\mathbf{u})=\mathbf{0}$ in region $A$ with boundary conditions $\mathbf{B}(\mathbf{u})=0$ on boundary $\partial A$, has a non-zero solution $\mathbf{u}$, the necessary and sufficient condition for the inhomogeneous system, $L(\mathbf{v})=\mathbf{b}$ in region $A$ with boundary conditions $\mathbf{B}(\mathbf{v})=\mathbf{0}$ on $\partial A$, to have a non-zero solution, is that the solvability condition, $\int_{\partial A} \mathbf{u} \cdot \mathbf{b} \mathrm{d} A=0$, must be satisfied.

The vibrational equations of a non-rotating open spherical shell, i.e. eqns (4) and (5) with $F^{\beta}=\rho h \omega^{2} u^{\beta}$ and $F^{3}=\rho h \omega^{2} w$, and boundary conditions (9) and (10) with any set of curvilinear coordinates chosen as independent variables can be proved to be self-adjoint (Hwang, 1993), which, in turn, implies the self-adjoint of the system of eqns (15) and the associated boundary conditions. The solvability condition, that is, the integration of the dot product of the vector composed of the right-hand side of eqn (16) and the vector $\left(\bar{u}_{1}, \bar{u}_{2}, \bar{w}\right)$ with the weight function $\sin \theta$ from $\theta=0$ to $\theta=\theta_{0}$ is zero, gives

$$
\begin{array}{r}
\int_{0}^{\theta_{0}}\left\{\frac{0}{\bar{u}_{1}}\left(-\omega_{0} \omega_{1} \stackrel{0}{\bar{u}}^{1}+\omega_{0}^{2} \frac{0}{\bar{v}} \frac{\cos \theta}{R}\right)+\stackrel{0}{\bar{u}}_{2}\left[\omega_{0}^{2}\left(\frac{0}{\bar{u}} \cos \theta+\frac{0}{\bar{w}} \sin \theta-\omega_{0} \omega_{1} \frac{0}{\bar{v}}\right) \frac{1}{R \sin \theta}\right]\right. \\
\left.+\frac{0}{\bar{w}}\left[\omega_{0}^{2} \frac{0}{\bar{v}} \sin \theta-\omega_{0} \omega_{1} \stackrel{0}{\bar{w}}\right]\right\} \sin \theta \mathrm{d} \theta=0
\end{array}
$$


where $\theta_{0}$ is the opening angle of the open shell, $\stackrel{0}{\bar{u}}, \bar{v}$, and $\stackrel{0}{\bar{w}}$ are the solution of equations (15) and (17a, c), and can be extracted from the exact solution (Niordson, 1984) listed in Appendix A. Using the orthonomal property

$$
\int_{0}^{\theta_{0}}\left[(\bar{u})^{2}+(\bar{v})^{2}+(\bar{w})^{2}\right] \sin \theta \mathrm{d} \theta=1,
$$

$\omega_{0}$ comes out from eqn (18) as

$$
\omega_{1}=2\left\{\int_{0}^{\theta_{0}}[\bar{u} \cos \theta+\bar{w} \sin \theta]^{0} \bar{v} \sin \theta \mathrm{d} \theta\right\} \omega_{0}=f(\mathbf{u}) \omega_{0} .
$$

Then the first approximation of the natural frequency of the $p$ th circumferential and $n$th latitudinally bending mode is

$$
\omega=\omega_{0}+\epsilon \omega_{1}=\omega_{0}+\Omega f(\mathbf{u})
$$

The frequencies of the forward and backward travelling waves are, respectively,

$$
\left|\omega_{+}\right|=\left|\omega_{0}\right|+\Omega f(\mathbf{u}), \quad\left|\omega_{-}\right|=\left|\omega_{0}\right|-\Omega f(\mathbf{u}) .
$$

The combination of these two travelling waves gives

$$
\cos \left(p \phi-\left|\omega_{+}\right| t\right)+\cos \left(p \phi+\left|\omega_{-}\right| t\right)=2 \cos \{p \phi-\Omega f(\mathbf{u}) t\} \cos \left|\omega_{0}\right| t .
$$

\section{Discussions}

The Coriolis force induced by the rotation of the shell will cause the natural frequency $\omega_{0}$ of the $(p, n)$ (the $p$ th circumferential and $n$th latitudinal) vibrational mode of the nonrotating shell to bifurcate into two, that is, $\left|\omega_{+}\right|$and $\left|\omega_{-}\right|$given by eqn (21). It also causes the vibrational mode to be split into two travelling waves which travel along the circumferential direction, the forward travelling wave with wave speed $\left|\omega_{+}\right|$less than $\omega_{0}$ and the backward travelling wave with wave speed $\left|\omega_{-}\right|$greater than $\omega_{0}$. As mentioned by Bryan (1980) for the rotating ring or cylindrical shell, these two travelling waves of same amplitude can be combined into a standing wave which moves relative to the rotating ring or cylindrical shell with negative angular velocity. The precessional phenomenon of vibrational mode is also found in open spherical shell and is used as the working principle of integrating gyros (Loper and Lynch, 1983). Equation (22) shows that the standing wave has the frequency $\left|\omega_{0}\right|$ which is the same as that of the non-rotating shell and moves relative to the shell with angular velocity $\Omega f(\mathbf{u}) / p$ or moves relative to an inertial space with a precessional rate $\Omega+\Omega f(\mathbf{u}) / p$, where $f(\mathbf{u})$ is negative as can be seen in the following example. This precessional rate is linearly proportional to the spin rate of the shell and becomes larger for higher circumferential mode.

A particular open spherical shell of thickness $h=0.1 \mathrm{~cm}$, radius $R=5 \mathrm{~cm}$, density $\rho=8 \mathrm{~g} \mathrm{~cm}^{-3}$, Young's modulus $E=2 \times 10^{11} \mathrm{NT} \mathrm{cm}^{-2}$, and Poisson ratio $v=0.3$ is considered. The frequencies obtained by theory (Niordson, 1985) are very close to those obtained experimentally by modal testing for the first bending $(n=1)$ and different circumferential $(p=2,3,4,5)$ modes as shown in Table 1. Equation (19) indicates that $f(\mathbf{u})$

Table 1. Natural frequencies $(\mathrm{Hz})$ by theoretical prediction and experimental measurement

\begin{tabular}{ccccc}
\hline$(p, n)$ & $(2,1)$ & $(3,1)$ & $(4,1)$ & $(5,1)$ \\
\hline Theory & 405 & 1101 & 2056 & 3229 \\
Experiment & 410 & 1086 & 2003 & 3125 \\
\hline
\end{tabular}


Table 2. Precessional angles of mode shapes for different $\theta_{0}$ when the shell rotates $90^{\circ}$

\begin{tabular}{llll}
\hline$(p, n)$ & $\theta_{0}=60^{\circ}$ & $0_{0}=75^{\circ}$ & $\theta_{0}=90^{\circ}$ \\
\hline$(2,1)$ & $76.3^{\circ}$ & $70.58^{\circ}$ & $65.07^{\circ}$ \\
$(3,1)$ & $81.77^{\circ}$ & $78.43^{\circ}$ & $75.4^{\circ}$ \\
$(4,1)$ & $84.58^{\circ}$ & $82.49^{\circ}$ & $80.71^{\circ}$ \\
\hline
\end{tabular}

Table 3. The value of $f(\mathbf{u})$ for the $(p, 2)$ modes, where $p=1,2, \ldots, 5$, for different values of Poisson's ratio

\begin{tabular}{cccccc}
\hline$(p, n)$ & $v=0.1$ & $v=0.2$ & $v=0.3$ & $v=0.4$ & $v=0.5$ \\
\hline$(2,2)$ & -0.28927 & -0.31413 & -0.33874 & -0.36319 & -0.38765 \\
$(3,2)$ & -0.26603 & -0.28706 & -0.30730 & -0.32675 & -0.34538 \\
$(4,2)$ & -0.24827 & -0.26849 & -0.28809 & -0.30702 & -0.32526 \\
$(5,2)$ & -0.22927 & -0.24849 & -0.26724 & -0.28549 & -0.30319 \\
\hline
\end{tabular}

depends on the opening angle $\theta_{0}$. Our numerical results show that the smaller the opening angle is, the larger is the precessional rate. When the shell rotates $90^{\circ}$, the precessional angle of nodal lines is listed in Table 2. For a hemispherical shell with the mode $(p, n)=(2$, 1), the precessional angle is $65.07^{\circ}$. A $63^{\circ}$ rotation of the standing wave was approximately measured by Loper and Lynch (1983) for $\theta_{0}$ lying in the range $95^{\circ}-96^{\circ}$. The $f(\mathbf{u})$ in eqn (19) depends on the vibrational displacements (or mode shape) $\stackrel{0}{\vec{u}}, \stackrel{0}{\vec{v}}, \stackrel{0}{\bar{w}}$ of the non-rotating hemispherical shell. From Appendix A we know that these displacements depend on the quantities $c_{u 1}, \ldots, c_{w 4}$ which, in turn, depend on the material property such as Poisson's ratio $v$ explicitly. Table 3 gives the values of $f(\mathbf{u})$ for the second latitudinal and various circumferential modes with different values of Poisson's ratio. Since the second decimal digit is varied, the effect of $v$ on $f(\mathbf{u})$ or the precession rate is significant for the moderate or high frequency modes such as the $(2,2)$ and $(3,2)$ modes. Although the present paper treats the spin rate as a constant, our results apply to the more general case of time-varying inertial rates as proven by Zhuravlev and Popov (1985) that the precession of the standing wave is an integrating phenomenon.

\section{THE EFFECT OF CENTRIFUGAL FORCE}

The centrifugal force which is neglected in the preceding sections is included here and its effect on the natural frequencies and precession rate are analysed. It is assumed that the strain caused by the centrifugal force is small. Linear strain-displacement and stress-strain relations allow us to decompose the displacements (or stress) into initial and incremental ones. The initial displacements (or stress) result from the non-vibrating shell experiencing centrifugal force. The incremental displacements (or stress) are caused by the vibration of the rotating shell and are measured relative to the initial state. The initial stress is approximated by the stress in the membrane state. The membrane equations of equilibrium for a rotating but non-vibrating shell are obtained by setting the components of moment tensor equaling zero (i.e. $M^{\alpha \beta}=0$ ) in eqns (4) and (5)

$$
\begin{aligned}
D_{\alpha} \bar{N}^{\alpha \beta}+\bar{F}^{\beta} & =0, \\
\bar{N}_{\alpha}^{\alpha} & =R \bar{P},
\end{aligned}
$$

where $\bar{N}^{\alpha \beta}$ is the initial membrane stress. The stress boundary conditions at the free edge are

$$
N=\bar{N}^{\alpha \beta} n_{\beta} n_{\alpha}=0, \quad S=\bar{N}^{\alpha \beta} n_{\beta} t_{\alpha}=0, \quad \text { at } \theta=\theta_{0} .
$$

Carrying out the covariant derivative, cqn (23a) becomes 


$$
\bar{N}_{, \alpha}^{\alpha \beta}+\bar{N}^{\gamma \beta} \Gamma_{\alpha \gamma}^{\alpha}+\bar{N}^{\alpha \gamma} \Gamma_{\alpha \gamma}^{\beta}+\bar{F}^{\beta}=0, \quad \beta=1,2 .
$$

The second one of eqn (25) is identically zero, because all the stresses are independent of the circumferential coordinate $\phi$ and the centrifugal force $\overline{\mathbf{F}}=\bar{F}^{\beta} \mathbf{a}_{\beta}+\bar{P}_{\mathbf{a}_{3}}$ has component $\bar{F}^{2}=0$. Since the centrifugal force is axisymmetric, shear stresses vanish, i.e. $\bar{N}^{12}=\bar{N}^{21}=0$. The resultant equations for unknowns $\bar{N}^{11}$ and $\bar{N}^{22}$ are

$$
\begin{aligned}
\bar{N}_{.1}^{11}+\bar{N}^{11} \Gamma_{12}^{2}+\bar{N}^{22} \Gamma_{22}^{1}+\bar{F}^{1} & =0, \\
\bar{N}_{1}^{1}+\bar{N}_{2}^{2} & =R P,
\end{aligned}
$$

where $\bar{F}^{1}=\rho h \Omega^{2} \sin \theta \cos \theta$ and $\bar{P}=\rho h \Omega^{2} \sin ^{2} \theta$. Using

$$
\bar{N}^{11}=a^{11} \bar{N}_{1}^{1}=\frac{1}{R^{2}} \bar{N}_{1}^{1}, \quad \bar{N}^{22}=\frac{1}{R^{2} \sin ^{2} \theta} \bar{N}_{2}^{2}, \quad \Gamma_{12}^{2}=\cot \theta, \Gamma_{22}^{1}=-\sin \theta \cos \theta,
$$

eqns (26) are simplified to

$$
\begin{aligned}
\left(\bar{N}_{1}^{1} \sin \theta\right)_{, \theta}-\cos \theta \bar{N}_{2}^{2}+\rho h \Omega^{2} R^{2} \sin ^{2} \theta \cos \theta & =0, \\
\bar{N}_{1}^{1}+\bar{N}_{2}^{2} & =\rho h \Omega^{2} R^{2} \sin ^{2} \theta .
\end{aligned}
$$

The solutions of eqn (27) give the initial membrane stresses:

$$
\bar{N}_{1}^{1}=0, \quad \bar{N}_{2}^{2}=\rho h \Omega^{2} R^{2} \sin ^{2} \theta
$$

The strain-stress relation,

$$
E_{\alpha \beta}=\frac{1}{E h}\left[(1+v) N_{\alpha \beta}-v a_{\alpha \beta} N_{\mu}^{\mu}\right]
$$

gives the initial strains

$$
E_{11}=-\frac{v}{E} \rho \Omega^{2} R^{4} \sin ^{2} \theta, \quad E_{22}=\frac{1}{E} \rho \Omega^{2} R^{4} \sin ^{4} \theta
$$

The strain-displacement equation (1) gives initial displacements

$$
\begin{aligned}
u_{1} & =\frac{1+v}{E} \rho \Omega^{2} R^{4} \sin \theta(\cos \theta-1), \\
u_{2} & =0 \\
w & =\frac{1}{E} \rho \Omega^{2} R^{3}\left[\sin ^{2} \theta-(1+v)\left(\cos ^{2} \theta-\cos \theta\right)\right] .
\end{aligned}
$$

The terms in equations of equilibrium, but neglected in the membrane equations, are the ignored bending stress denoted by $E\left(\bar{M}_{\alpha}^{\alpha}\right)$ and

$$
E\left(\bar{M}_{\alpha}^{\alpha}\right)=D_{\alpha} D_{\beta} \bar{M}^{\alpha \beta}+\frac{1}{R^{2}} \bar{M}_{\alpha}^{\alpha}
$$

The initial bending stress $\bar{M}^{\alpha \beta}$ can be calculated by substituting eqn (31) into eqn (7). Then the ratio of $E\left(\bar{M}_{\alpha}^{\alpha}\right)$ to $\bar{P}$ can be evaluated as 


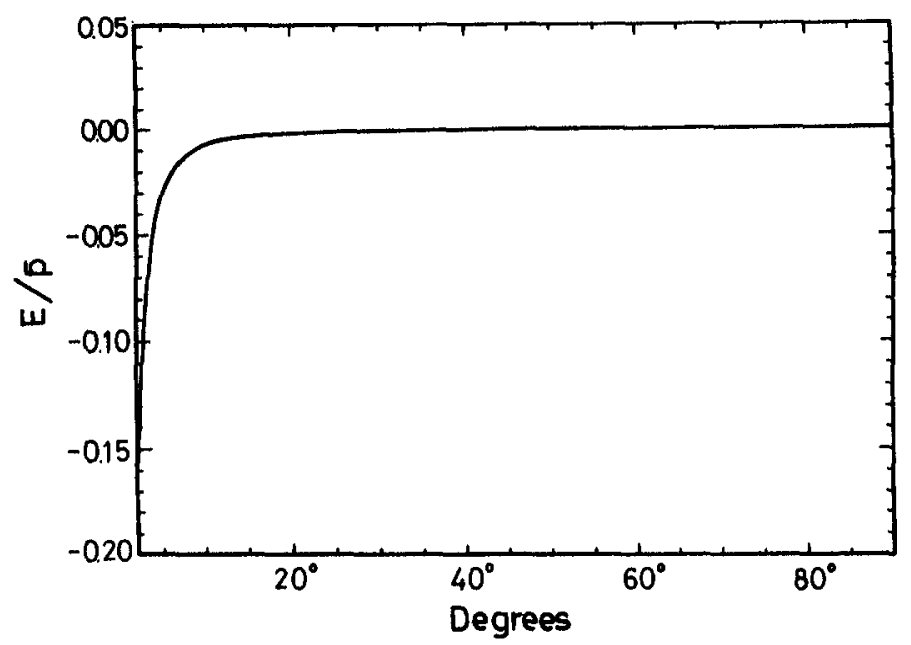

Fig. 2. The plot of $E\left(\bar{M}_{\alpha}^{\alpha}\right) / \bar{P}$ versus latitudinal coordinate $\theta$.

$$
\frac{E\left(\bar{M}_{\alpha}^{\alpha}\right)}{\bar{P}}=\frac{(h / R)^{2}}{6\left(1-v^{2}\right)}\left\{\left[(v+1)^{2}+4\right]-[(3+v)(1+v)+8] \cot ^{2} \theta\right\}
$$

The plot of $E\left(\bar{M}_{\alpha}^{\alpha}\right) / \bar{P}$ versus latitudinal coordinate $\theta$ for $h / R=0.01$ and $v=0.3$ is shown in Fig. 2. The result indicates that the ignored initial bending stress outside the neighborhood of $\theta=0$ is close to zero. So the approximation of the actual initial state by the membrane state is justified.

\section{Incremental equations of motion}

The equations of motion of a rotating shell for the incremental state are derived in the invariant form in this section based on the skeleton of Niordson shell theory. In order to include the effect of initial stresses some terms in the strain-displacement equation that has been neglected in the linearized equation (1) must be retained (Soedel, 1981). The full strain-displacement equation is

$$
\begin{aligned}
E_{\alpha \beta}=\frac{1}{2}\left(D_{\alpha} u_{\beta}+D_{\beta} u_{\alpha}\right)-d_{\alpha \beta} w+ & \frac{1}{2}\left[D_{\alpha} u^{\gamma} D_{\beta} u_{\gamma}-d_{\beta \gamma} w D_{\alpha} u^{\gamma}-d_{\alpha \gamma} w D_{\beta} u^{\gamma}\right. \\
& \left.+d_{\alpha \gamma} d_{\beta}^{\gamma}(w)^{2}+d_{\alpha \gamma} d_{\beta \delta} u^{\gamma} u^{\delta}+d_{\alpha \gamma} u^{\gamma} w_{, \beta}+d_{\beta \gamma} u^{\gamma} w_{, \alpha}+w_{, \alpha} w_{, \beta}\right]
\end{aligned}
$$

Since in-plane deformation is small compared to the transverse one, we neglect the square terms of in-plane deformation and the product terms of the in-plane and transverse deformation in the non-linear part of strain-displacement equations. Then the incremental membrane strain has the form

$$
E_{\alpha \beta}=E_{\alpha \beta}^{\mathrm{L}}+E_{\alpha \beta}^{\mathrm{N}}
$$

where $E_{\alpha \beta}^{\mathrm{L}}$ and $E_{\alpha \beta}^{\mathrm{N}}$ denote respectively the linear and non-linear part of the strain and they are

$$
\begin{aligned}
& E_{\alpha \beta}^{\mathrm{L}}=\frac{1}{2}\left(D_{\alpha} u_{\beta}+D_{\beta} u_{\alpha}\right)-d_{\alpha \beta} w, \\
& E_{\alpha \beta}^{\mathrm{N}}={ }_{2}^{1}\left[d_{\alpha \gamma} d_{\beta}^{\gamma}(w)^{2}+w_{, \alpha} w_{, \beta}\right] .
\end{aligned}
$$

The total membrane strain $N^{* \alpha \beta}$ of the shell is expressed as 


$$
N^{* \alpha \beta}=\bar{N}^{\alpha \beta}+N^{\alpha \beta},
$$

where $\bar{N}^{\alpha \beta}$ and $N^{\alpha \beta}$ are the initial and incremental stresses, respectively. The principle of virtual work appears in the following form

$$
\begin{aligned}
\int_{\mathscr{\alpha}}\left[N^{* \alpha \beta} \delta E_{\alpha \beta}\right. & \left.+M^{\alpha \beta} \delta K_{\alpha \beta}\right] \mathrm{d} A=\int_{\mathscr{A}}\left[F^{* \alpha} \delta u_{\alpha}+P^{*} \delta w\right] \mathrm{d} A \\
& +\oint_{\mathscr{L}}\left[T^{\alpha} \delta u_{\alpha}+Q \delta w+M_{B} \frac{\partial}{\partial n} \delta w\right] \mathrm{d} s+\oint_{\mathscr{L}_{0}}\left[T^{\alpha} \delta u_{\alpha}+Q \delta w+M_{B} \frac{\partial}{\partial n} \delta w\right] \mathrm{d} s,
\end{aligned}
$$

where $\mathscr{A}$ denote the area of the middle surface of the shell, $F^{* \alpha}$ and $P^{*}$ are the in-plane and transverse body forces, respectively, $\mathscr{L}_{0}$ is the boundary curve of the middle surface at the clamped edage if the clamped angle is considered infinitesimal instead of zero, $T^{x}$ are the in-plane boundary forces, and $Q$ and $M_{B}$ are the effective shear force and bending moment on the boundary as defined in eqn (10). In the left-hand side of eqn (37), those terms, which will contribute to the non-linear part of the incremental equations of motion, are dropped. Then the remaining linear terms are

$$
\text { left-hand side of eqn (37) }=\int_{\mathscr{\alpha}}\left[\bar{N}^{\alpha \beta} \delta E_{\alpha \beta}^{\mathrm{N}}+N^{* \alpha \beta} \delta E_{\alpha \beta}^{\mathrm{L}}+M^{\alpha \beta} \delta K_{\alpha \beta}\right] \mathrm{d} A \text {. }
$$

The variation of stresses in each term of the left-hand side of eqn (37) is expressed in terms of variation of displacements in the following and integration by parts is used if it is needed,

$$
\begin{aligned}
& \int_{\mathscr{\alpha}} \bar{N}^{\alpha \beta} \delta E_{\alpha \beta}^{\mathrm{N}} \mathrm{d} A=\frac{1}{2} \int_{\mathscr{\alpha}}\left[2 \bar{N}^{\alpha \beta} d_{\alpha \gamma} d_{\beta}^{\gamma} w \delta w+\bar{N}^{\alpha \beta}\left(w_{, \alpha} \delta w_{, \beta}+w_{, \beta} \delta w_{, \alpha}\right)\right] \mathrm{d} A \\
& =\int_{\mathscr{\alpha}}\left[\bar{N}^{\alpha \beta} d_{\alpha \gamma} d_{\beta}^{y} w-D_{\beta}\left(\bar{N}^{\alpha \beta} w_{, \alpha}\right)\right] \delta w \mathrm{~d} A+\oint_{\mathscr{L}}\left(\bar{N}^{\alpha \beta}{ }_{, \alpha} n_{\beta}\right) \delta w \mathrm{~d} s+\oint_{\mathscr{L}_{0}}\left(\bar{N}^{\alpha \beta} w_{, \alpha} n_{\beta}\right) \delta w \mathrm{~d} s, \\
& \int_{\mathscr{\alpha}} N^{* \alpha \beta} \delta E_{\alpha \beta}^{\mathrm{L}} \mathrm{d} A=-\int_{\mathscr{\alpha}}\left[D_{\alpha} N^{* \alpha \beta} \delta u_{\beta}+d_{\alpha \beta} N^{* \alpha \beta} \delta w\right] \mathrm{d} A \\
& +\oint_{\mathscr{L}} N^{* \alpha \beta} n_{\beta} \delta u_{x} \mathrm{~d} s+\oint_{\mathscr{L}_{0}} N^{* \alpha \beta} n_{\beta} \delta u_{\alpha} \mathrm{d} s \\
& \int_{\mathscr{A}} M^{\alpha \beta} \delta K_{\alpha \beta} \mathrm{d} A=\int_{\mathscr{A}}\left\{\left(D_{\beta} D_{\alpha} M^{\alpha \beta}-d_{\beta \gamma} d_{\alpha}^{\gamma} M^{\alpha \beta}\right) \delta w\right. \\
& \left.+\delta u_{\gamma}\left(-M^{\alpha \beta} D_{\beta} d_{\alpha}^{\gamma}-2 d_{\alpha}^{\gamma} D_{\beta} M^{\alpha \beta}\right)\right\} \mathrm{d} A
\end{aligned}
$$

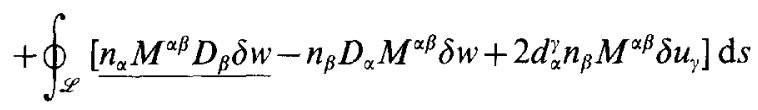

$$
\begin{aligned}
& +\oint_{\mathscr{L}_{0}}\left[n_{\alpha} M^{\alpha \beta} D_{\beta} \delta w-n_{\beta} D_{\alpha} M^{\alpha \beta} \delta w+2 d_{\alpha}^{\prime \prime} n_{\beta} M^{\alpha \beta} \delta u_{\gamma}\right] \mathrm{d} s,
\end{aligned}
$$

where the equation

$$
\delta K_{\alpha \beta}=D_{\alpha} D_{\beta} \delta w+d_{\alpha \gamma} D_{\beta} \delta v^{\gamma}+d_{\beta \gamma} D_{\alpha} \delta v^{\gamma}+\delta v^{\gamma} D_{\beta} d_{\gamma \alpha}-d_{\beta \gamma} d_{\alpha}^{\gamma} \delta w
$$

and the equation of Codazzi $D_{\alpha} d_{\beta \gamma}-D_{\beta} d_{\alpha y}=0$ have been used.

Using the following formula 


$$
\oint_{\mathscr{L}} B^{\alpha} \phi_{, \alpha} \mathrm{d} s=\oint_{\mathscr{L}} B^{\alpha} n_{\alpha} \frac{\partial \phi}{\partial n} \mathrm{~d} s-\oint_{\mathscr{L}} \phi \frac{\partial}{\partial s}\left(B^{\alpha} t_{\alpha}\right) \mathrm{d} s,
$$

the underlined terms in the right-hand side of eqn (38d) can be converted to the form

$$
\oint_{\mathscr{L}} n_{\alpha} M^{\alpha \beta} D_{\beta} \delta w \mathrm{~d} s=\oint_{\mathscr{L}} M^{\alpha \beta} n_{\alpha} n_{\beta} \frac{\partial \delta w}{\partial n} \mathrm{~d} s-\oint_{\mathscr{L}} \delta w \frac{\partial}{\partial s}\left(M^{\alpha \beta} n_{\alpha} t_{\beta}\right) \mathrm{d} s .
$$

Substituting eqn (38) into eqn (37), letting the coefficients of $\delta u_{\alpha}(\alpha=1,2)$ and $\delta w$ in the area integral equal zero since $\delta u_{\alpha}, \delta w$ are independent, $\delta u_{\alpha}=\delta w=\partial \delta w / \partial n=0$ in the line integral along $\mathscr{L}_{0}$ since variation of displacements at the clamped edge is not allowed, and also letting the coefficients of $\delta u_{\alpha}, \delta w$, and $\partial \delta w / \partial n$ in the line integral along $\mathscr{L}$ equal zero, we obtain the linear equations of motion

$$
\begin{aligned}
D_{\beta} N^{* \alpha \beta}+M^{\gamma \beta} D_{\beta} d_{\gamma}^{\alpha}+2 d_{\gamma}^{\alpha} D_{\beta} M^{\gamma \beta}+F^{* \alpha}=0, \\
D_{\beta} D_{\alpha} M^{\alpha \beta}-d_{\alpha \gamma} d_{\beta}^{\gamma} M^{\alpha \beta}-d_{\alpha \beta} N^{* \alpha \beta}+\bar{N}^{\alpha \beta} d_{x \gamma} d_{\beta}^{\gamma} w \quad D_{\beta}\left(\bar{N}^{\alpha \beta} w_{, \alpha}\right)=P^{*},
\end{aligned}
$$

the boundary conditions for the clamped edge

$$
u=v=w=\frac{\partial w}{\partial \theta}=0
$$

and the stress boundary conditions at the free edge $\left(\theta=\theta_{0}\right)$

$$
\begin{aligned}
N & =\left(N^{* \alpha \beta}+2 d_{\gamma}^{\alpha} M^{\beta \gamma}\right) n_{\alpha} n_{\beta}=0, \\
S & =\left(N^{* \alpha \beta}+2 d_{\gamma}^{\alpha} M^{\beta \gamma}\right) n_{\alpha} t_{\beta}=0, \\
M_{B} & =M^{\alpha \beta} n_{\alpha} n_{\beta}=0, \\
Q & =\bar{N}^{\alpha \beta} w_{, \alpha} n_{\beta}-n_{\beta} D_{\alpha} M^{\alpha \beta}-\frac{\partial}{\partial S}\left(M^{\alpha \beta} n_{\alpha} t_{\beta}\right)=0 .
\end{aligned}
$$

The alternative bending tensor for the incremental state-like eqn (3) is

$$
\tilde{K}_{\alpha \beta}=K_{\alpha \beta}+\frac{2}{R} E_{\alpha \beta}^{\mathbf{L}}
$$

By using the principle of virtual

$$
\bar{N}^{\alpha \beta} \delta E_{\alpha \beta}^{\mathrm{N}}+N^{* \alpha \beta} \delta E_{\alpha \beta}^{\mathrm{L}}+M^{\alpha \beta} \delta K_{\alpha \beta}=\bar{N}^{\alpha \beta} \delta E_{\alpha \beta}^{\mathrm{N}}+\tilde{N}^{* \alpha \beta} \delta E_{\alpha \beta}^{\mathrm{L}}+M^{\alpha \beta} \delta \widetilde{K}_{\alpha \beta},
$$

the appropriate membrane stress tensor is obtained as $\tilde{N}^{* \alpha \beta}=N^{* \alpha \beta}-(2 / R) M^{\alpha \beta}$. Being expressed in terms of $\tilde{N}^{* \alpha \beta}$ instead of $N^{* \alpha \beta}$ and using $d_{\alpha \beta}=-a_{\alpha \beta} / R$, eqns (39) and (40b) become

$$
\begin{aligned}
D_{\alpha} \tilde{N}^{* \alpha \beta}+F^{* \beta} & =0 \\
D_{\alpha} D_{\beta} M^{\alpha \beta}+\frac{1}{R^{2}} M_{\alpha}^{\alpha}+\frac{1}{R} \bar{N}_{\alpha}^{* \alpha}+\frac{w}{R^{2}} \bar{N}_{\alpha}^{\alpha}-D_{\beta}\left(\tilde{N}^{\alpha \beta} w_{, \alpha}\right) & =P^{*},
\end{aligned}
$$


and

$$
\begin{aligned}
N & =\tilde{N}^{* \alpha \beta} n_{\alpha} n_{\beta}=0, \quad S=\tilde{N}^{* \alpha \beta} n_{\alpha} t_{\beta}=0, \\
M_{B} & =M^{\alpha \beta} n_{\alpha} n_{\beta}=0, \quad Q=\bar{N}^{\alpha \beta} w_{. \alpha} n_{\beta}-n_{\beta} D_{\alpha} M^{\alpha \beta}-\frac{\partial}{\partial s}\left(M^{\alpha \beta} n_{\alpha} t_{\beta}\right)=0 .
\end{aligned}
$$

Substracting eqn (23) from eqn (43) gives the incremental equations of motion

$$
\begin{aligned}
D_{\alpha} \tilde{N}^{\alpha \beta}+F^{\beta} & =0, \\
D_{\alpha} D_{\beta} M^{\alpha \beta}+\frac{1}{R^{2}} M_{\alpha}^{\alpha}+\frac{1}{R} \tilde{N}_{\alpha}^{\alpha}+\frac{w}{R^{2}} \bar{N}_{\alpha}^{\alpha}-D_{\beta}\left(\bar{N}^{\alpha \beta} w_{. \alpha}\right) & =P .
\end{aligned}
$$

It is noted that in eqn (23) $\bar{F}^{i}=-\rho h\left[\mathbf{\Omega} \times\left(\boldsymbol{\Omega} \times \mathbf{u}^{\text {ini }}\right)\right] \cdot \mathbf{a}^{i}\left(i=1,2,3\right.$ with $\left.\bar{F}^{3}=\bar{P}\right)$ where $\mathbf{u}^{\text {ini }}$ is the initial displacement and $\ddot{\mathbf{u}}^{\text {ini }}=\dot{\mathbf{u}}^{\text {ini }}=0$, and in eqn (43) $F^{* i}=-\rho h\{\ddot{\mathbf{u}}+2[\boldsymbol{\Omega} \times \dot{\mathbf{u}}]$ $\left.+\boldsymbol{\Omega} \times\left[\mathbf{\Omega} \times\left(\mathbf{u}^{\text {ini }}+\mathbf{u}\right)\right]\right\} \cdot \mathbf{a}^{i}\left(i=1,2,3\right.$ with $\left.F^{* 3}=P^{*}\right)$ where $\mathbf{u}$ is denoted as the incremental displacement and $\left(\mathbf{u}^{\mathrm{ini}}+\mathbf{u}\right)$ is the total displacement. The components of body force in eqn (45) is

$$
F^{i}=F^{* i}-\bar{F}^{i}=-\rho h[\ddot{\mathbf{u}}+2(\boldsymbol{\Omega} \times \dot{\mathbf{u}})+\mathbf{\Omega} \times(\mathbf{\Omega} \times \mathbf{u})] \cdot \mathbf{a}^{i}, \quad i=1,2,3
$$

with $F^{3}=P$. The subtraction of eqn (24) from eqn (44) gives the stress boundary conditions at the free edge for the incremental state

$$
\begin{aligned}
N & =\tilde{N}^{\alpha \beta} n_{\alpha} n_{\beta}=0, \quad S=\tilde{N}^{\alpha \beta} n_{\alpha} t_{\beta}=0, \\
M_{B} & =M^{\alpha \beta} n_{\alpha} n_{\beta}=0, \quad Q=\bar{N}^{\alpha \beta} w_{, \alpha} n_{\beta}-n_{\beta} D_{\alpha} M^{\alpha \beta}-\frac{\partial}{\partial s}\left(M^{\alpha \beta} n_{\alpha} t_{\beta}\right)=0 .
\end{aligned}
$$

The solutions of eqn (45) for the incremental displacements are expressed in the form of travelling waves like eqn (11). For the $p$ th circumferental mode they are

$$
\begin{aligned}
u_{1}(\theta, \phi, t) & =\bar{u}_{1}(\theta) \cos (p \phi-\omega t), \\
u_{2} & =\bar{u}_{2}(\theta) \sin (p \phi-\omega t), \\
w(\theta, \phi, t) & =\bar{w}(\theta) \cos (p \phi-\omega t) .
\end{aligned}
$$

The substitution of eqn (48) into eqn (45) gives

$$
\begin{array}{r}
\overline{L^{1}(\mathbf{u})+\rho h \omega^{2} u^{1}}-2 \rho h \Omega \omega \bar{v} \frac{\cos \theta}{R}-\rho h \frac{\Omega^{2}}{R}\left[\bar{w} \sin \theta \cos \theta+\bar{u} \cos ^{2} \theta\right]=0, \\
\frac{L^{2}(\mathbf{u})+\rho h \omega^{2} u^{2}}{2}-2 \rho h \omega \frac{\Omega}{R}[\bar{u} \cot \theta+\bar{w}]-\rho h \frac{\Omega^{2}}{R} \frac{\bar{v}}{\sin \theta}=0, \\
\overline{L(\mathbf{u})+\rho h \omega^{2} w}-2 \rho h \Omega \omega \bar{v} \sin \theta+\rho h \Omega^{2}\left[p^{2} \bar{w}-\bar{u} \sin \theta \cos \theta\right]=0 .
\end{array}
$$

The substitution of eqn (48) into eqn (46) yields

$$
\begin{aligned}
\bar{N} & =\overline{\tilde{N}^{\alpha \beta} n_{\alpha} n_{\beta}}=0, \quad \bar{S}=\overline{\overline{N^{\alpha \beta} n_{\alpha} t_{\beta}}=0,} \\
\bar{M}_{B} & =\overline{M^{\alpha \beta} n_{\alpha} n_{\beta}}=0, \quad \bar{Q}=-\overline{n_{\beta} D_{\alpha} M^{\alpha \beta}-\frac{\partial}{\partial s}\left(M^{\alpha \beta} n_{\alpha} t_{\beta}\right)}=0 .
\end{aligned}
$$

Again the overlined quantities in eqns (48), (49), and (50) mean that they are functions of $\theta$ only. In the previous case where only the Coriolis force is considered, the first-order 
perturbed frequencies of the rotating shell are obtained by solving the solvability condition without actually finding out the perturbed displacements such as $u^{\alpha}$ and $w$ in eqn (13). The effect of centrifugal force on the natural frcquency will appear in the second-order perturbed term in eqn (13c). Thus the first-order perturbed system, i.e. eqn (16), must be solved prior to solving the second-order perturbed frequency. An alternative approach, the Galerkin method and frequency perturbation, will be adopted to solve the natural frequency and to avoid the tedious manipulations for solving the perturbed displacements. In the application of the Galerkin technique, the latitudinal mode $\overline{\mathbf{u}}(\theta)$ in eqn (48) of the $p$ th circumferentially travelling wave is expanded in series of the eigenfunctions $\mathbf{u}_{m}(m=1,2, \ldots)$ which are the latitudinal mode shape of the non-rotating open shell at the $p$ th circumferential wave. That is,

$$
\overline{\mathbf{u}}(\theta)=\sum_{m} A_{m} \overline{\mathbf{u}}_{m}(\theta)=\sum_{m} A_{m}\left\{\begin{array}{c}
\bar{u}_{m}(\theta) \\
\bar{v}_{m}(\theta) \\
\bar{w}_{m}(\theta)
\end{array}\right\}
$$

Let $\omega_{\mathrm{m}}$ denote the eigenvalue (or natural frequency) corresponding to the eigenfunction $\mathbf{u}_{\mathrm{m}}$. The substitution of eqn (51) into eqn (49a) gives the residual $R_{1}$ as

$$
R_{1}=\sum_{m} \rho h\left(\omega^{2}-\omega_{m}^{2}\right) A_{m} \bar{u}_{m}-\rho h \sum_{m} A_{m}\left[2 \omega \Omega \bar{v}_{m} \cos \theta-\Omega^{2}\left(\bar{w}_{m} \sin \theta \cos \theta+\bar{u}_{m} \cos ^{2} \theta\right)\right] .
$$

Similarly, substituting eqn (51) into eqns (49b) and (49c) yields

$$
\begin{aligned}
& R_{2}=\sum_{m} \rho h\left(\omega^{2}-\omega_{m}^{2}\right) A_{m} \bar{v}_{m}-\rho h \sum_{m} A_{m}\left[2 \omega \Omega\left(\bar{u}_{m} \cos \theta+\bar{w}_{m} \sin \theta\right)-\Omega^{2} \bar{v}_{m}\right], \\
& R_{3}=\sum_{m} \rho h\left(\omega^{2}-\omega_{m}^{2}\right) A_{m} \bar{w}_{m}-\rho h \sum_{m} A_{m}\left[2 \omega \Omega \bar{v}_{m} \sin \theta+\Omega^{2}\left(p^{2} \bar{w}_{m}-\bar{u}_{m} \sin \theta \cos \theta\right)\right] .
\end{aligned}
$$

Taking the inner product of the residual vector $\left(R_{1}, R_{2}, R_{3}\right)$ and the $n$th latitudinal mode shape $\left(\bar{u}_{n}, \bar{v}_{n}, \bar{w}_{n}\right)$ and integrating them over the area of the middle surface, we have

$$
\begin{aligned}
& \sum_{m} \rho h\left(\omega^{2}-\omega_{m}^{2}\right) A_{m} \int\left(\bar{u}_{m} \bar{u}_{n}+\bar{v}_{m} \bar{v}_{n}+\bar{w}_{m} \bar{w}_{n}\right) \sin \theta \mathrm{d} \theta \\
& =2 \rho h \omega \Omega \sum_{m} A_{m} \int\left[\left(\bar{u}_{m} \bar{v}_{n}+\bar{u}_{n} \bar{v}_{m}\right) \cos \theta+\left(\bar{v}_{m} \bar{w}_{n}+\bar{v}_{n} \bar{w}_{m}\right) \sin \theta\right] \sin \theta \mathrm{d} \theta \\
& +\rho h \Omega^{2} \sum_{m} A_{m} \int\left[p^{2} \bar{w}_{m} \bar{w}_{n}-\bar{u}_{m} \bar{u}_{n} \cos ^{2} \theta-\bar{v}_{m} \bar{v}_{n}-\left(\bar{w}_{m} \bar{u}_{n}+\bar{w}_{n} \bar{u}_{m}\right) \sin \theta \cos \theta\right] \sin \theta \mathrm{d} \theta .
\end{aligned}
$$

Defining

$$
G_{m n}=\int\left[\left(\bar{u}_{m} \bar{v}_{n}+\bar{u}_{n} \bar{v}_{m}\right) \cos \theta+\left(\bar{v}_{m} \bar{w}_{n}+\bar{v}_{n} \bar{w}_{m}\right) \sin \theta\right] \sin \theta \mathrm{d} \theta
$$

and

$$
H_{m n}=\int\left[p^{2} \bar{w}_{m} \bar{w}_{n}-\bar{u}_{m} \bar{u}_{n} \cos ^{2} \theta-\bar{v}_{m} \bar{v}_{n}-\left(\bar{w}_{m} \bar{u}_{n}+\bar{w}_{n} \bar{u}_{m}\right) \sin \theta \cos \theta\right] \sin \theta \mathrm{d} \theta
$$

and using the orthonomal property of the eigenfunctions $\mathbf{u}_{m}$, eqn (53) can be simplified to 


$$
A_{n}\left(\omega^{2}-\omega_{n}^{2}\right)=2 \omega \Omega \sum_{m} A_{m} G_{m n}+\Omega^{2} \sum_{m} A_{m} H_{m n}, \quad n=1,2, \ldots
$$

The non-dimensional wave speeds of the travelling waves in the rotating shell and the nondimensional natural frequencies of the non-rotating shell are defined as

$$
\bar{\omega}=\frac{\omega}{\omega_{1}}, \quad \bar{\omega}_{n}=\frac{\omega_{n}}{\omega_{1}}, \quad n=1,2, \ldots
$$

where $\left|\omega_{1}\right|$ is the natural frequency of the first latitudinal and $p$ th circumferential vibrational mode of the non-rotating shell. The notation $\epsilon$ is used again but now is defined as the small ratio of the spin rate $\Omega$ to $\omega_{1}$, i.e. $\epsilon=\Omega / \omega_{1}$. Equation (56) can be rewritten as

$$
A_{n}\left(\bar{\omega}^{2}-\bar{\omega}_{n}^{2}\right)=2 \bar{\omega} \epsilon \sum_{m} A_{m} G_{m n}+\epsilon^{2} \sum_{m} A_{m} H_{m n}, \quad n=1,2, \ldots
$$

or in matrix form

$$
[\mathrm{D}] \mathbf{A}=\mathbf{0},
$$

where $\mathbf{A}=\left(A_{1}, A_{2}, \ldots, A_{n}\right)^{\mathrm{T}}$ and the element $D_{m n}$ of the matrix [D] is

$$
D_{m n}=-\left(\bar{\omega}^{2}-\bar{\omega}_{n}^{2}\right) \delta_{m n}+2 \epsilon \bar{\omega} G_{m n}+\epsilon^{2} H_{m n} .
$$

The wave speed is expanded in series of $\epsilon$ as follows:

$$
\bar{\omega}=\stackrel{0}{\bar{\omega}}+\epsilon \frac{1}{\bar{\omega}}+\epsilon^{2} \frac{2}{\bar{\omega}}+\cdots
$$

Substituting eqn (58) into eqn (57a) and letting the determinant of the matrix [D] equal zero yields

$$
\epsilon^{0} \mathscr{D}_{0}+\epsilon^{1} \mathscr{D}_{1}+\epsilon^{2} \mathscr{D}_{2}+\cdots=0
$$

Letting the coefficients of like powers of $\epsilon$ in the left-hand side of eqn (59) equal zero, we have

$$
\begin{aligned}
& \mathscr{D}_{0}=\prod_{m}\left(\bar{\omega}^{2}-\bar{\omega}_{m}^{2}\right)=0, \\
& \mathscr{D}_{1}=\left[\prod_{m}\left(\stackrel{0}{\bar{\omega}}^{2}-\bar{\omega}_{m}^{2}\right)\right] \times\left[\sum_{m}\left(\frac{2 \stackrel{0}{\bar{\omega}} G_{m m}-2 \stackrel{0}{\bar{\omega}} \frac{1}{\bar{\omega}}}{\stackrel{0}{\omega}^{2}-\bar{\omega}_{m}^{2}}\right)\right]=0,
\end{aligned}
$$

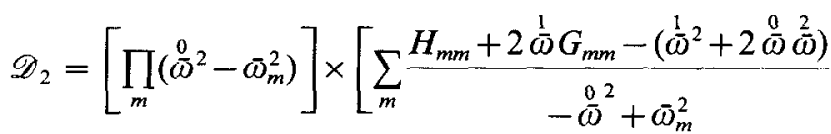

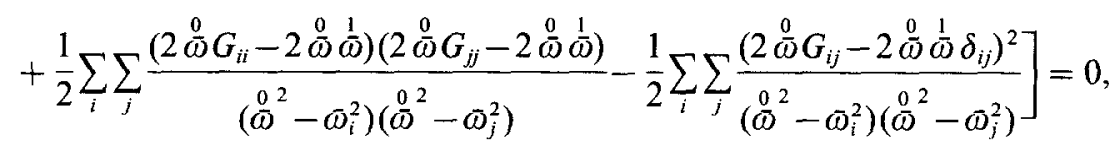


where the operator $\Pi$ is defined as

$$
\prod_{m=1}^{k} y_{m}=y_{1} y_{2} y_{3} \ldots y_{k}
$$

If $n$ finite terms are chosen in eqn (51), the $n$ roots of eqn (60) give the $n$ solutions of $\stackrel{0}{\omega}$. There are

$$
\stackrel{0}{\bar{\omega}}=\bar{\omega}_{1}, \bar{\omega}_{2}, \bar{\omega}_{3}, \ldots, \bar{\omega}_{n},
$$

their absolute values are the non-dimensional natural frequencies of the non-rotating shell. Substituting the $\frac{0}{\bar{\omega}}$ in eqn (63) into eqn (61) gives the $n$ solutions of $\frac{1}{\bar{\omega}}$

$$
\frac{1}{\bar{\omega}}=G_{k k}=2 \int_{0}^{\theta_{0}}\left[\bar{u}_{k} \cos \theta+\bar{w}_{k} \sin \theta\right] \bar{v}_{k} \sin \theta \mathrm{d} \theta, \quad k=1,2, \ldots, n
$$

which is the same as the function $f(\mathbf{u})$ in eqn (19). Substituting the obtained $\stackrel{0}{\mathscr{O}}$ and $\frac{1}{\mathscr{O}}$ into eqn (62) gives the $n$ solutions of $\stackrel{2}{\bar{\omega}}$

$$
\frac{2}{\bar{\omega}}=\frac{1}{2 \bar{\omega}_{k}}\left[\sum_{\substack{j=1 \\ j \neq k}}^{n} \frac{4 \bar{\omega}_{k}^{2} G_{k j}^{2}}{\bar{\omega}_{k}^{2}-\bar{\omega}_{j}^{2}}+G_{k k}^{2}+H_{k k}\right], \quad k=1,2, \ldots, n .
$$

The substitution of eqns (63), (64), and (65) into eqn (58) gives the wave speed of the $p$ th circumferential travelling wave in the rotating shell vibrating at the $k$ th latitudinally bending mode as

$$
\omega=\omega_{k}+\Omega G_{k k}+\frac{\Omega^{2}}{2 \omega_{k}}\left[\sum_{\substack{j=1 \\ j \neq k}}^{n} \frac{4 \omega_{k}^{2} G_{k j}^{2}}{\omega_{k}^{2}-\omega_{j}^{2}}+G_{k k}^{2}+H_{k k}\right]
$$

The natural frequency of the forward travelling wave having $k$ th latitudinally vibrational mode is

$$
\left|\omega_{+}\right|=\left|\omega_{k}\right|+\Omega G_{k k}+\frac{\Omega^{2}}{2\left|\omega_{k}\right|}\left[\sum_{\substack{j=1 \\ j \neq k}} \frac{4 \omega_{k}^{2} G_{k j}^{2}}{\omega_{k}^{2}-\omega_{j}^{2}}+G_{k k}^{2}+H_{k k}\right],
$$

and the natural frequency of the backward travelling wave having $k$ th latitudinally vibrational mode is

$$
\left|\omega_{-}\right|=\left|\omega_{k}\right|-\Omega G_{k k}+\frac{\Omega^{2}}{2\left|\omega_{k}\right|}\left[\sum_{\substack{j=1 \\ j \neq k}} \frac{4 \omega_{k}^{2} G_{k j}^{2}}{\omega_{k}^{2}-\omega_{j}^{2}}+G_{k k}^{2}+H_{k k}\right] .
$$

The second-order term in eqn (58), which appears in the terms proportional to $\Omega^{2}$ in eqns (67) and (68), is due to the effect of the centrifugal force and results in the increase of the natural frequencies of these two travelling waves. If this second-order term is neglected, the natural frequencies of travelling waves given by eqns (67) and (68) are exactly the same as those given by eqn (21). It is noteworthy that the second-order term in eqn (58) raises the vibrational frequency of the precessional standing wave which is the combination of the 


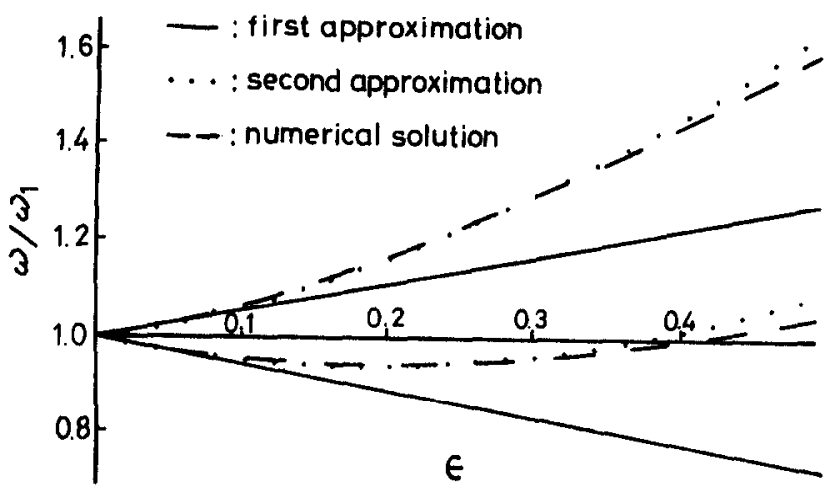

Fig. 3. The natural frequencies of forward and backward travelling waves of the $(p, n)=(2,1)$ mode versus $\epsilon\left(=\Omega / \omega_{1}\right)$.

two travelling waves in the same manner as eqn (22) in the previous section, but does not affect the precession rate.

\section{Discussion}

A particular shell considered in the previous section is used again here as an example. The natural frequencies of forward and backward second circumferential $(p=2)$ travelling waves with first latitudinal bending mode versus the small parameter $\epsilon$ are plotted in Fig. 3. In the approximation of the Galerkin method only the first three terms in the right-hand side of eqn (51) are taken into account. The values of coefficients $G_{i j}$ and $H_{i j}$ are listed in Tables 4 and 5, respectively. The solid lines in Fig. 3 indicate the frequencies given either by eqn (21) or by the first approximation of eqn (58) with the effect of the centrifugal force removed. The dotted lines indicate the frequencies given by the second approximation of eqn (58) with the effect of the centrifugal force considered. The dashed lines are the frequencies obtained by numerically solving the characteristic equation $\operatorname{det}[\mathrm{D}]=0$ resulting from eqn (57a). Although both first-order and second-order analytical solutions give the same result for the precession rate of the vibrational mode, for the natural frequency prediction, however, it is clear that, when the spin rate is slightly larger, e.g. in the range $0.1<\epsilon=\Omega / \omega_{1}<0.4$, the second-order solution is more accurate than the first-order one.

\section{CONCLUSIONS}

The linear relation between the precession rate of the vibrational mode (or the standing wave) and the spin rate of the rotating open spherical shell is the basic principle by which a hemispherical resonator gyro predicts the angular rate by measuring the precessional

Table 4. Coefficient $G_{i j}$ for a shell of open angle $90^{\circ}$,

$$
G_{i j}=G_{j i}
$$

\begin{tabular}{cccc}
\hline$G_{i j}$ & 1 & 2 & 3 \\
\hline 1 & -0.5548 & -0.0523 & -0.0057 \\
2 & - & -0.3387 & -0.0171 \\
3 & - & - & -0.1355 \\
\hline
\end{tabular}

Table 5. Coefficient $H_{i j}$ for a spherical shell of open angle $90^{\circ}, H_{i j}=H_{j i}$

\begin{tabular}{cccc}
\hline$H_{i j}$ & 1 & 2 & 3 \\
\hline 1 & 2.7202 & 0.3925 & 0.0786 \\
2 & - & 2.6619 & 0.5694 \\
3 & - & - & 2.7784 \\
\hline
\end{tabular}


angle of the vibrational mode. In the present paper, the analytical expression for the precession rate is obtained so that the linear relation can be determined quantitatively. It is found that the factor which causes the precession of the standing waves is the Coriolis force; the centrifugal force only affects the vibrational frequencies of the standing waves. Poisson's ratio has significant influence on the precession rate especially for moderate or high frequency modes.

\section{REFERENCES}

Bryan, G. H. (1980). On the beats in the vibrations of a revolving cylinder or bell. Proc. Cambridge Philos. Soc. VII, 101-111.

Beckemeyer, R. J. and Eversman, W. (1971). The free vibrations of a spinning centrally clamped shallow spherical shell. J. Appl. Mech. 93, 601-607.

Carrier, G. F. (1945). On the vibration of the rotating ring. Q. Appl. Math. 3, 235-245.

Chen, Y., Zhan, H. B., Shen, Z. P., Grieger, I. and Kroplin, B. H. (1993). Vibrations of high speed rotating shells with calculations for cylindrical shells. J. Sound Vibration 160(1), 137-160.

Egarmin, N. E. (1986). Precession of vibrational standing waves of a rotating axisymmetric Shell. Izv. AN SSSR, Mekhanika Tverdogo Tela 21(1), 142-148.

Endo, M., Hatamura, K., Sakata, M. and Taniguchi, O (1984). Flexural vibration of a thin rotating ring. J. Sound Vibration $92(2), 261-272$.

Huang, S. C. and Soedel, W. (1988). On the forced vibration of simply supported rotating cylindrical shells. $J$. Accoust. Soc. Am. 84(1), 275-285.

Hwang, J. J. (1993). Dynamical analysis of a hemispherical resonantor gyroscope. Ph.D. Dissertation, National Taiwan University, Taipei, P.R. China, pp. 106-110.

Kainins, A. and Wilkinson, J. P. (1965). On nonsymmetric dynamics problems of elastic shells. J. Appl. Mech. 32, 525-532.

Loper, E. I. and Lynch, D. D. (1983). Projected system performance based on recent HRG test results. Proc. IEEE/AIAA 5th Digital Avionics Systems Conf., 31 October-3 November, Seattle, WA.

Love, A. E. H. (1888). The small free vibrations and deformations of a thin elastic shell. Phil. Trans. R. Soc, Ser. $A, 495-546$.

Matthews, A. and Rybak, F. J. (1992). Comparison of hemispherical resonator gyro and optical gyros. IEEE AES Mag. 7, 40-46.

Niordson, F. I. (1985). Shell Theory. North-Holland.

Niordson, F. I. (1984). Free vibrations of thin elastic spherical shells. Int. J. Solids Structures 20 (7), 667-687.

Saito, T. and Endo, M. (1986). Vibration of finite length, rotating cylindrical shells. J. Sound Vibration 107 (1), 17-28.

Shevchuk, G. J. and Thullen, P. (1978). Flexural vibrations of rotating electromagnetic shields. ASME J. Mech. Des. 101, 133-137.

Soedel, W. (1981). Vibrations of Shells and Plates. Marcel Dekker.

Srinivasan, A. V. and Lauterbach, G. F. (1971). Traveling waves in rotating cylindrical shells. J. Eng. Ind. ASME 93, 1229-1232.

Zhuravlev, V. F. and Klimov, D. M. (1983). Dynamic effects in an elastic rotating ring. Izv. AN SSSR. Mekhanika Tverdogo Tela 18(5), 17-23.

Zhuravlev, V. F. and Popov, A. L. (1985). Precession of the natural vibration mode of a spherical shell upon rotation. Izv. AN SSSR. Mekhanika Tverdogo Tela 20(1), 147-151.

\section{APPENDIX A}

The displacements $u, v$, and $w$ of a non-rotating vibrational shell for the $m$ th circumferential mode are

$$
\begin{aligned}
& u=\frac{1}{R^{2}}\left(\sum_{i=1}^{4} c_{u i} A_{i}\right) \cos m \phi, \\
& v=\frac{1}{R^{2}}\left(\sum_{i=1}^{4} c_{v i} A_{i}\right) \sin m \phi, \\
& w=\frac{1}{R^{2}}\left(\sum_{i=1}^{4} c_{w i} A_{i}\right) \cos m \phi,
\end{aligned}
$$

where $A_{i}(i=1, \ldots, 4)$ are determined by the boundary conditions (Niordson, 1984),

$$
\begin{aligned}
c_{u 1} & =(1+v)\left(\mathscr{P}_{1}-m \cot \theta \mathscr{P}\right), & c_{u 2} & =(1+v)\left(\mathscr{R}_{1}-m \cot \theta \mathscr{R}\right), \\
c_{u 3} & =(1+v)\left(\mathscr{P}_{1}-m \cot \theta \mathscr{P}\right), & c_{u 4} & =-\frac{m}{\sin \theta} \mathscr{Q}, \\
c_{v 1} & =-(1+v) \frac{m}{\sin \theta} \mathscr{P}, & c_{t 2} & =-(1+v) \frac{m}{\sin \theta} \mathscr{R},
\end{aligned}
$$




$$
c_{\imath 3}=-(1+v) \frac{m}{\sin \theta} \mathscr{P}, \quad c_{v 4}=-m \cot \theta \mathscr{Q}+\mathscr{Q}_{1},
$$

and

$$
\begin{array}{ll}
c_{w 1}=-a_{4} \mathscr{P}, & c_{w 2}=-a_{6} \mathscr{R}-\eta \mathscr{S}, \\
c_{w 3}=-a_{6} \mathscr{S}+\eta \mathscr{R}, & c_{w 4}=0 .
\end{array}
$$

The $\mathscr{P}, \mathscr{P}_{1}, \mathscr{Q}, \mathscr{Q}_{1}, \mathscr{R}, \mathscr{R}_{1}, \mathscr{S}$, and $\mathscr{S}_{1}$ are the associated Legendre functions

$$
\begin{array}{ll}
\mathscr{P}=P_{\sigma}^{-m}(\cos \theta), & \mathscr{P}_{1}=P_{\sigma}^{-m+1}(\cos \theta), \\
\mathscr{Q}=P_{\tau}^{-m}(\cos \theta), & \mathscr{Q}_{1}=P_{\tau}^{-m+1}(\cos \theta), \\
\mathscr{R}=R_{\zeta}^{-m}(\cos \theta), & \mathscr{R}_{1}=R_{\zeta}^{-m+1}(\cos \theta), \\
\mathscr{P}=S_{\zeta}^{-m}(\cos \theta), & \mathscr{P}_{1}=S_{\zeta}^{-m+1}(\cos \theta),
\end{array}
$$

where $R_{\zeta}^{-m}$ and $S_{\zeta}^{-m}$ are the real and imaginary parts of $P_{\zeta}^{-m}$ if $\zeta$ is complex. The $a_{4}$ and $a_{6}$ in the expressions for $c_{w 1}, c_{w 2}$, and $c_{w: 3}$ are defined by

$$
a_{4}=\lambda+1-v-\beta, \quad a_{6}=\lambda+1-v-\xi .
$$

The degrees (subscripts) $\sigma, \zeta$, and $\tau$ of the above-mentioned associated Legendre functions are related to $\beta, \xi, \eta$. and $\rho$ by equations

$$
\sigma(\sigma+1)=-\beta, \quad \zeta(\zeta+1)=-\xi-\mathrm{i} \eta, \quad \tau(\tau+1)=-\rho .
$$

The $\beta, \xi$, and $\eta$ are the coefficients of the differential equation for a vibrating spherical shell

$$
\left(\Delta-\frac{\beta}{R^{2}}\right)\left(\Delta-\frac{\xi+\mathrm{i} \eta}{R^{2}}\right)\left(\Delta-\frac{\xi-\mathrm{i} \eta}{R^{2}}\right) \mathscr{E}=0,
$$

where $\mathscr{E}$ is a potential function. 